Thorax (1950), 5, 233.

\title{
REDUCTION IN BRONCHIAL SUBDIVISION IN BRONCHIECTASIS
}

\author{
BY \\ LYNNE McA. REID* \\ From the Royal Melbourne Hospital
}

\begin{abstract}
Bronchiectasis, as the derivation of the word implies, is the name given to any condition in which there is dilatation of one or more bronchi. Since it is clear that increase in the diameter of a bronchus may result from a variety of conditions, among them infections and massive collapse, the term describes an anatomical abnormality rather than a single disease. $\mathrm{A}$ wide range exists both in the form and degree of the dilatation and in its distribution among the bronchi. As the reason for the diversity is not clearly understood, it is not possible at present to make a satisfactory classification of the miscellaneous conditions which are all grouped together as bronchiectasis. In this, as in any other disease, accurate study of the pathological anatomy is an essential step in its elucidation. But, although the abnormal morphology of the dilated bronchi is the outstanding feature, it has been imperfectly described.

During the examination of the large number of bronchograms and specimens seen in the routine work of a chest unit, it was noticed that the number of subdivisions of the bronchi between the hilum and the periphery of the lung differed from case to case, and was frequently much smaller than in the normal lung. It was decided to investigate this apparent reduction, and, in the event of its being demonstrated, to determine the fate of the missing subdivisions. This paper is a report on the findings.
\end{abstract}

\section{Selection and Preparation of the Material}

Forty-five cases of lobectomy for bronchiectasis, selected because well-preserved specimens and clear bronchograms were available, form the basis of this paper. In all of them the bronchiectasis was the main feature. Cases in which it was secondary to some other condition, such as chronic lung abscess or carcinoma, were excluded. Normal lungs from three patients who died from non-pulmonary disease were examined as controls.

Within two hours of removal at operation the lobe was inflated by way of the bronchial tree with $8 \%$ formol-saline, until it was approximately the size and shape it had been in the body. Because of leakage, specimens from segmental dissections did not inflate as well as those from cases in which the whole lobe had been removed, but as far as possible an even distribution of fixative was obtained. The specimens were kept in formalin for at least five and usually 10 days before being cut, as by this time fixation was even and complete and the specimens maintained their shape after cutting.

\footnotetext{
* Research Fellow, National Health and Medical Research Council.
} 


\section{Method of Examination}

To simplify the technical work one broncho-pulmonary segment was selected for examination, and a count was made of the number of subdivisions of the bronchial tree in this part of the lung. With two exceptions the segment chosen was the posterior basal (Brock, 1946) of the lower lobe (right or left), because this was most frequently involved in the bronchiectatic process. The two exceptions were cases of saccular bronchiectasis involving only the right middle lobe.

Counts of the number of generations of the bronchial tree were made using the following methods: (1) Study of the bronchogram ; (2) macroscopic dissection of the excised specimen without any means of magnification; (3) examination of serial microscopic sections cut at right angles to the long axis of the bronchus.

In counting the subdivisions the posterior basal branch of the lower lobe bronchus was counted as the first generation, the branches arising from its first division the second generation, and so on down its length. Where it was possible, the count was extended to, but did not include, the respiratory bronchioles. These could be demonstrated in the normal material, but in the pathological specimens it was usually not possible to trace the bronchial tree as far as structures which could be recognized as respiratory bronchioles.

It was found that side branches leading to the costal surface of the posterior basal segment reached their destination with fewer subdivisions than those passing to the diaphragm. Because of this variation, the maximum number of bifurcations between the centre and the periphery was estimated in each specimen, so that the figures would be comparable. In counting, therefore, the branch judged to be most nearly axial was traced to the next division, and where such identification was difficult, the one passing towards the diaphragmatic surface was chosen.

\section{StUdY OF THE BRONCHOGRAM}

A count made of the subdivisions of the bronchial tree visible in the bronchogram is probably always only approximate, even in the normal lung, because of the frequent difficulty in identifying all the branches of any given bronchus; this difficulty arises partly from overlying shadows of bronchi in neighbouring lung tissue. Alveolar filling, if excessive, further increases the difficulty by masking subdivisions. The ease of counting depends on adequate delineation of the bronchial tree without excessive filling and requires films of technical excellence, which ensure vivid contrast. Oblique and lateral films are often more helpful than postero-anterior views but, ideally, comparison of films taken in different directions should be made.

There are also problems peculiar to the different types of bronchial dilatation. In cases of "cylindrical" bronchiectasis, in which the proximal part of the bronchial tree is well filled, any confusion in tracing the successive side branches of a bronchus is usually caused by overlying bronchi. In the "saccular" type on occasion it is difficult to be certain of continuity between the balloon-like areas of lipiodol. Where the outlines of the bronchi are irregular, expansions of the bronchial lumen which resemble diverticula may be mistaken for side branches.

Experience in correlating the form of the bronchi in the bronchogram with that seen in the specimens was helpful in this perplexity. In the normal arrangement 
it was seen that the first five or six divisions arose approximately $1 \mathrm{~cm}$. apart and that the angle which separated them from the continuation of the parent stem was usually less than a right angle. Beyond these the branches arose more closely together and passed laterally at approximately a right angle. Although the bronchial branching seen in bronchograms of "cylindrical" bronchiectasis is decreased, it follows the normal pattern. In the more irregular types confusion was occasionally caused by side expansions whose length was usually less than the width of their opening into the axial bronchus, from which they passed at approximately a right angle, while true side branches were generally longer and were separated from the axial bronchus by less than a right angle.

Although it was frequently impossible to ascertain with certainty all the successive subdivisions of a given bronchus some constancy was achieved in the counts. The variation between estimations of the bronchial subdivisions made on different occasions was only two or three. As other methods usually yielded higher counts and the maximum was selected for further comparison, the inaccuracy of the bronchogram was of no consequence in this investigation. Nor does it preclude use of the bronchogram by the clinician as an indication of the effect of the disease on bronchial subdivision, since, as will be shown below, there is some correlation between the counts made from the bronchogram and those made from the specimens.

\section{Examination of the Macroscopic Specimen}

In examining the macroscopic specimen a probe was inserted into the posterior basal bronchus and the lobe was divided in two by cutting along this probe. In this way the bronchus with its first three or four side branches was shown in longitudinal section. The more peripheral branches were laid open by cutting along their length with scissors, and removing surrounding lung tissue where this interfered with satisfactory exposure. Where there was sclerosis of the lung tissue and considerable distortion of the bronchi, their ramifications were difficult to trace. In the "cylindrical" type it was usually possible to follow the bronchial tree to its finer branchings but in the other types only proximal subdivisions could be demonstrated.

\section{Histological Examination}

Serial sections were cut at right angles to the long axis of the bronchus. In the normal lungs and the first pathological specimens examined, these were prepared about 0.6 to $1.0 \mathrm{~mm}$. apart, right from the beginning of the posterior basal bronchus, but as no submacroscopic branches were demonstrated in this way over the proximal part of the bronchus, in which divisions were obvious macroscopically, blocks were subsequently prepared from only the peripheral part. In cases of "cylindrical" bronchiectasis these started at about the tenth generation and in the other types from about $1 \mathrm{~cm}$. proximal to the macroscopic termination of the bronchus.

The blocks were usually about $2 \mathrm{~cm} . \times 3 \mathrm{~cm}$. in area and $1 \mathrm{~cm}$. thick. Sections were cut $7 \mu$ thick and about every seventieth section mounted so that successive sections were about $500 \mu$ apart. Haematoxylin-eosin was used as the stain for this examination, but in examining the sections to identify remnants of the bronchi special stains were used. These included Van Gieson's, Masson's, Verhoeff's, Mayer's mucicarmine, and Weigert's elastin stain. 


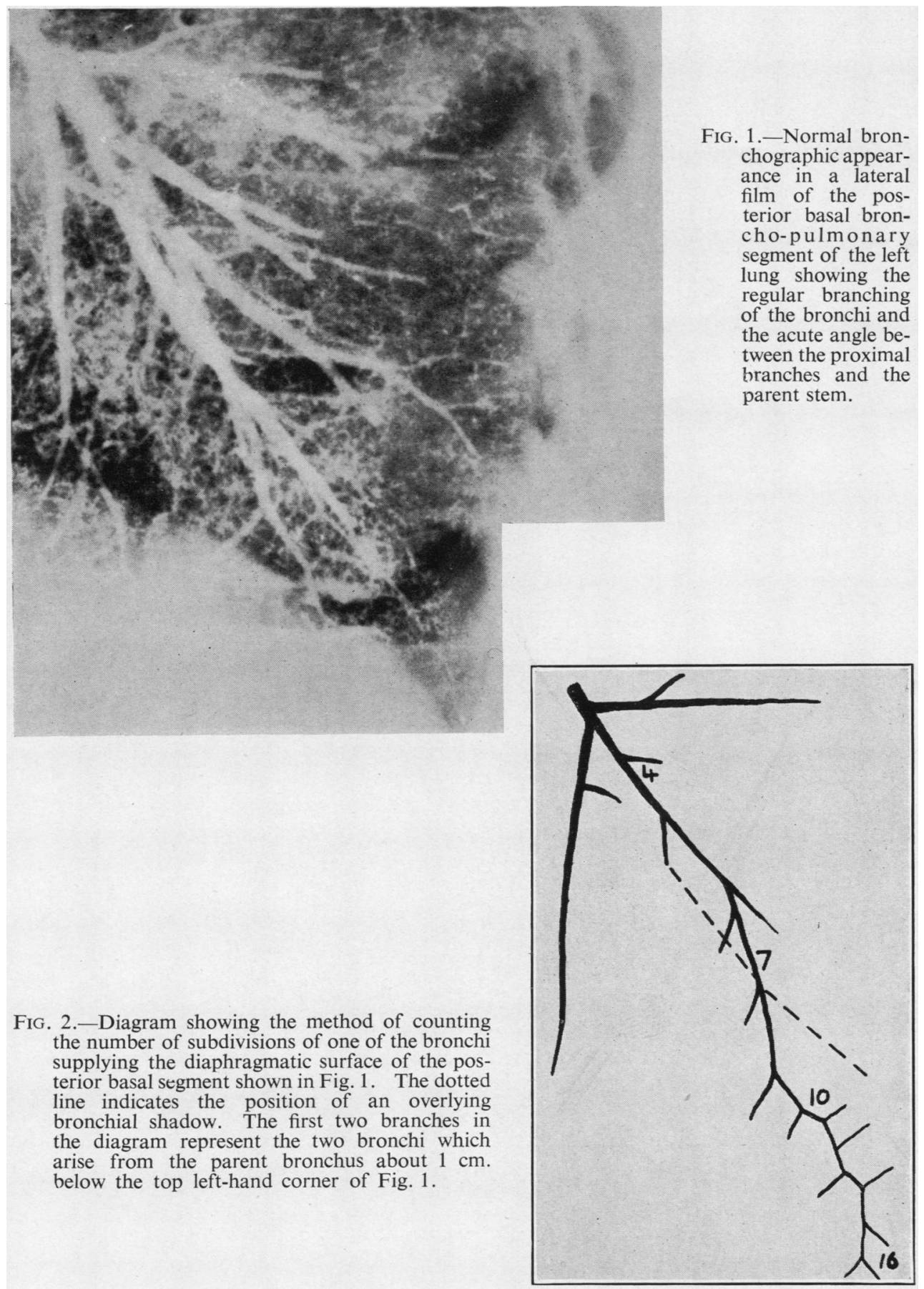
ance in a latera film of the posung showing the of the bronchi and the acute angle between the proximal branches and the parent stem. the number of subdivisions of one of the bronchi supplying the diaphragmatic surface of the posbasal segment shown in Fig. 1. The dotted bronchial shadow. The first two branches in the diagram represent the two bronchi which arise from the parent bronchus about $1 \mathrm{~cm}$. below the top left-hand corner of Fig. 1. 
To examine the whole field simultaneously and thus facilitate counting the number of branches a magnification of only 12 was used. Higher magnification was used in identification of the finer bronchioles and of structural remnants of diseased bronchi. As the bronchus was traced a diagram was prepared of the progressive divisions seen and the maximum count was then taken.

\section{The Normal Lung}

In the posterior basal bronchi of the three normal specimens, macroscopic dissection showed the average maximum number of subdivisions to be 17 ; and on microscopic examination this was increased to 20 . This is the number usually demonstrable in well-defined normal bronchograms (Fig. 1).

Estimations of the number of generations present in the normal bronchial tree have been made by several workers whose main concern was with the development of the mammalian and particularly the human lung. Using the middle lobe of an infant, Broman (1923) has estimated that the terminal outgrowth of the bronchial tree present at birth represents the eighteenth in a series of dichotomies, counting the main bronchus to the lung as the first generation. Palmer (1936) in his counts used the bronchus to the upper lobe and found 17 generations present at birth and up to 20 in the adult lung. The controversy between different authorities over lateral or terminal budding as the mechanism of postnatal development of the human lung does not affect the general agreement that the number of bronchial divisions present in the adult lung lies somewhere about 20 . If this be modified so that the bronchus to the posterior broncho-pulmonary segment is counted as the first generation (the method used here) the number will be about 16 or 17 (Fig. 2). As this is in accord with the findings in the three normal lungs examined in this investigation, this small number of controls was considered adequate.

As a preliminary to presenting the observations made on the pathological specimens the way in which they were grouped will be indicated, as this will make description of them clearer.

\section{Classification of the Pathological Specimens}

In examining the bronchograms two groups separated themselves rather clearly: the "saccular" type, in which there were usually only three or four generations of the bronchial tree ; and the "cylindrical" type, in which considerably more bronchi were present. Between these was a motley group in which the bronchi were intermediate in number. On this evidence the cases were divided into the three following groups. Their relation to the variety of descriptive names used by different authorities will be mentioned in the discussion.

Group I.-The bronchograms of cases in the first group show the "cylindrical" type of bronchiectasis (Fig. 3). This name well describes the bronchogram, in which the bronchi show a regular outline, no great increase in diameter, and usually end squarely and abruptly. The bronchogram has a "stripped" appearance as the bronchial walls are straight and the finer bronchi and bronchioles do not fill with lipiodol.

Group 1I.-In the second group the bronchi show in bronchograms as tubes of no regular form or size, dilated but deformed by sites of relative constriction. 


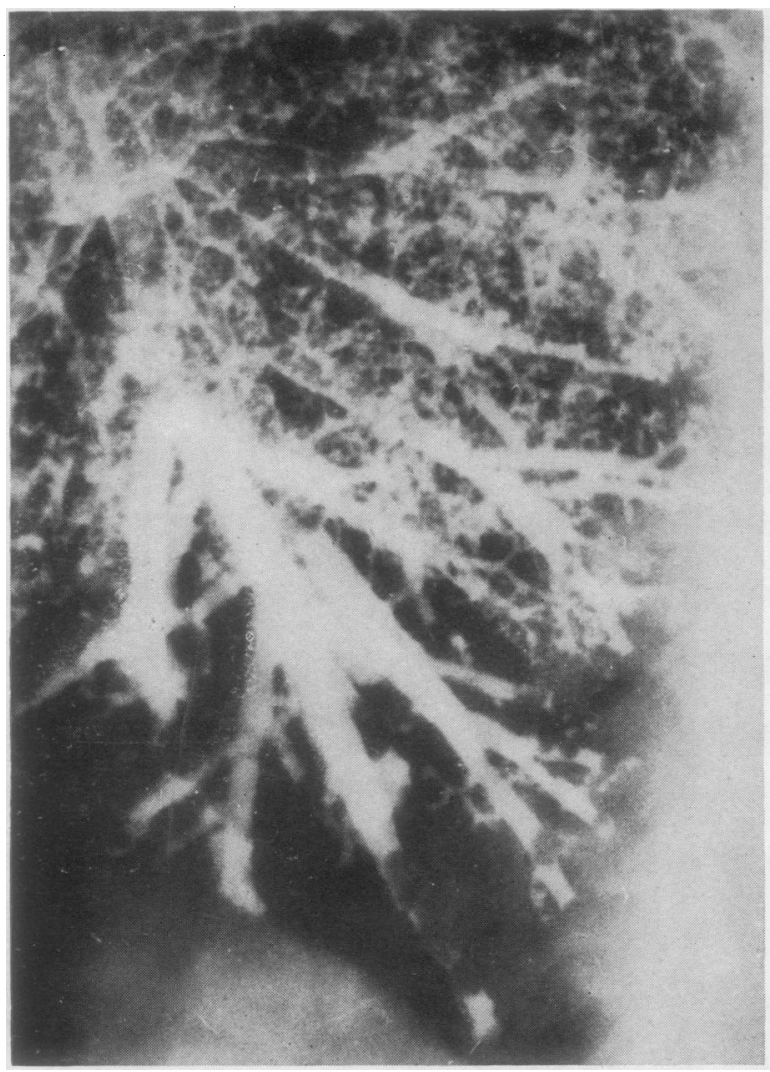

FIG. 3.-Lateral bronchographic view of "cylindrical" bronchiectasis in the posterior and middle basal bronchi of the left lower lobe. Nine or ten subdivisions of the posterior basal bronchus can be counted.
Occasionally they may be smooth in outline, but they are then different from a normal bronchus in that they do not show progressive reduction in diameter as they pass to the periphery, and also they have a bulbous and distorted end. There is, typically, no bronchiolar or alveolar filling. Irregularity of the bronchial outline and the bulbous termination are the cardinal features in the morphology of this group, in contrast to the straight outline and abrupt termination seen in the first group. The diameter of the bronchi is sometimes little greater than that seen in a corresponding generation of the bronchial tree in a normal lung. The term "varicose" well describes the form of many of the bronchi in this group, as they resemble in their distortion and irregular bulging the condition seen in veins, to which the adjective is more usually applied (Fig. 4).

Group III.-In the third group dilatation, increasing progressively towards the periphery of the lung, is the most prominent feature. The bronchus has a ballooned cutline, and although it can usually be traced almost to the pleura, only about four subdivisions can be seen. This is the commonly recognized "saccular" type.

As there is greater diversity of form in the intermediate group than is seen in cases from the other groups, more of the former have been included in this investigation. Only five of the "saccular" group were examined, as its members show little variation.

\section{The Pathological Specimens}

The results of counting the bronchial subdivisions by the three methods are shown in Table I. It is not felt that there is anything magical or absolute in the average figure of any group, but they are tabulated so that the difference between the groups will be more apparent. 
The first group shows most discrepancy between the counts obtained by the different methods. In the bronchograms an average of about seven bronchial subdivisions could be seen; more could be demonstrated by dissection, and more still by microscopic examination. (Fig. 5). In serial sections in five cases the average number of subdivisions which could be demonstrated was 16, which for the purpose of this discussion will be considered to be within normal limits.

In contrast to this was the third group, in which no more subdivisions of the bronchial tree could be seen microscopically than in the bronchogram. This was the lowest number present in any group, as the maximum number of divisions which could be counted was five (Figs. 8 and 9).

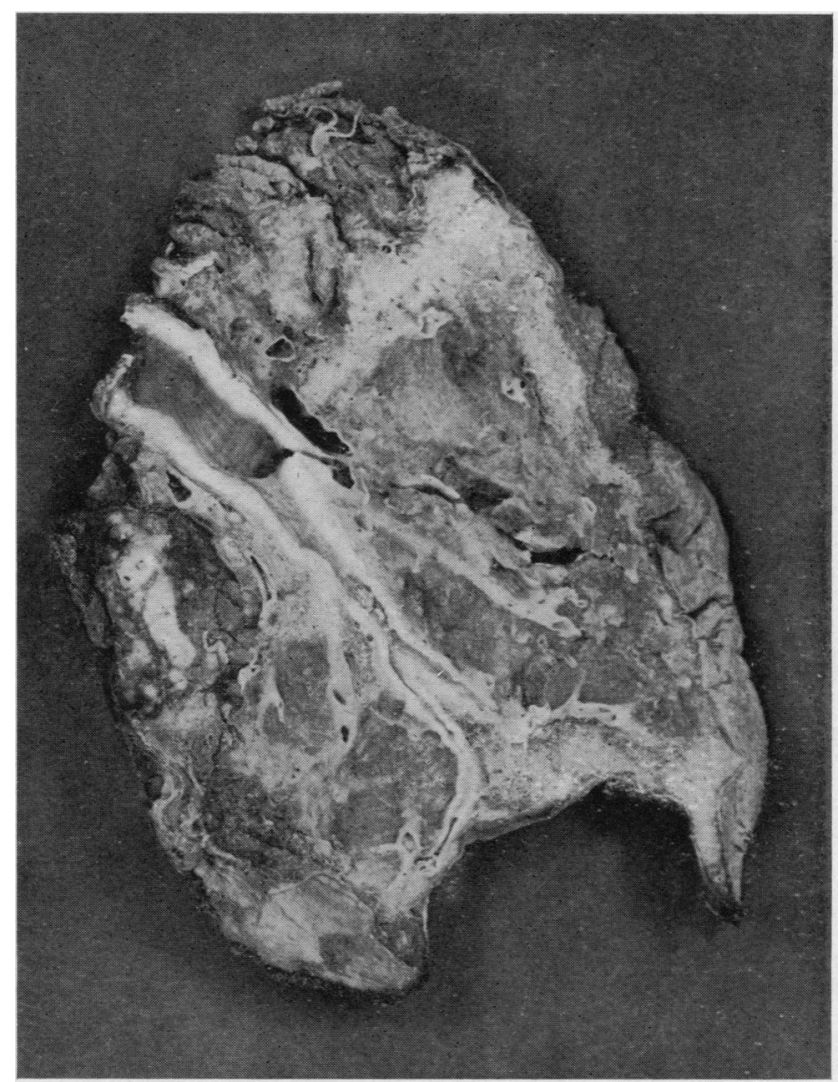

FIG. 4.-Operation specimen of "cylindrical" bronchiectasis to show the regular arrangement of the bronchial side branches and the gradual decrease in diameter of the bronchus.

Intermediate counts were obtained in the second group (Figs. 6 and 7). Here the radiograph often shows very little more of the bronchial tree than is seen in the "saccular" type, but microscopically, on an average, twice as many branches can be seen. There is wider variation between members of this group. Some show a characteristic of the third group in that microscopically no more bronchi can be demonstrated than are seen in the radiograph. In others dissection and microscopy each show further bronchial divisions.

In summary, then, the "cylindrical" group is the only one in which the full number of generations of the bronchial tree persists, although the peripheral branches of these bronchi do not fill with lipiodol on bronchography. In all the other types the number is reduced, the greatest reduction being in the "saccular" type.

\section{FATE OF THE "Missing Bronchi"}

Dissection of the specimens in the first group throws some light on the disparity between the number of bronchi seen in the bronchogram and on direct examination 


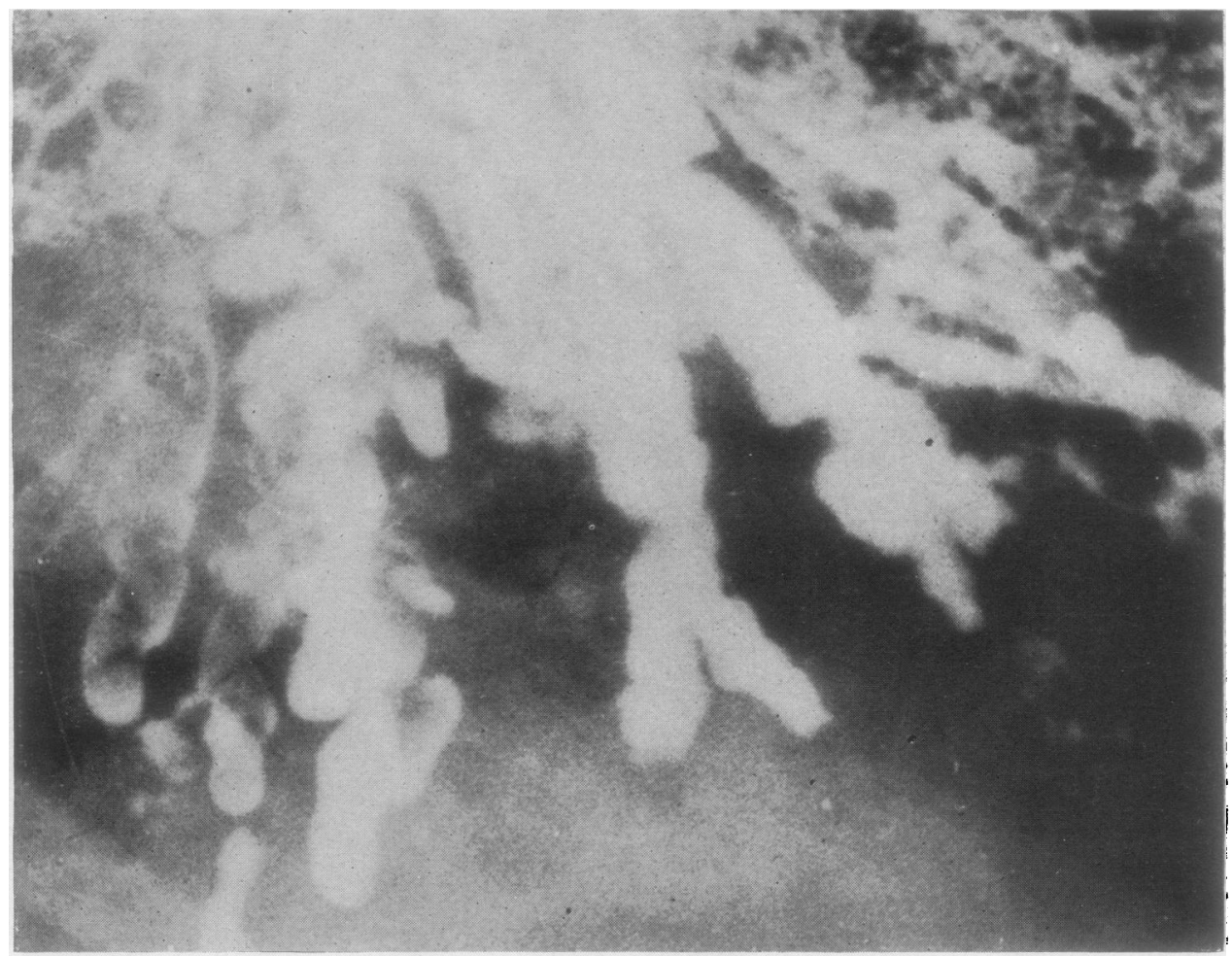

FIG. 5.-Antero-posterior view of bronchogram showing bronchiectasis cf the left true lower and dorsal lobes. This case shows the irregular "varicose" outline and rounded termination characteristic of the second group. A maximum of five generations of the posterior basal bronchus. could be counted from this and oblique films.

TABLE I

The Number of Bronchial Subdivisions in a Broncho-pulmonary Segment in CaSes OF BRONCHIECTASIS

\begin{tabular}{|c|c|c|c|c|c|c|}
\hline & & & \multirow{2}{*}{$\begin{array}{c}\text { Number } \\
\text { of } \\
\text { Cases }\end{array}$} & \multicolumn{3}{|c|}{ Average Number of Bronchial Divisions } \\
\hline & & & & Bronchogram & $\begin{array}{l}\text { Macroscopic } \\
\text { Specimen }\end{array}$ & $\begin{array}{c}\text { Microscopic } \\
\text { Serial Sections }\end{array}$ \\
\hline $\begin{array}{l}\text { Group I } \\
\text { "Cylindrical " }\end{array}$ & . & .. & 12 & $7.5(6-10)$ & $11(8-15)$ & $\begin{array}{l}16(15-18) \\
5 \text { cases }\end{array}$ \\
\hline $\begin{array}{l}\text { Group II } \\
\text { "Varicose" }\end{array}$ & & . & 28 & $4(2-8)$ & $6.5(3-12)$ & $\begin{array}{l}8(3-11) \\
16 \text { cases }\end{array}$ \\
\hline $\begin{array}{l}\text { Group III } \\
\text { "Saccular" }\end{array}$ & .. & .. & 5 & $3.5(3-4)$ & $4(3-4)$ & $\begin{array}{l}4(3-5) \\
5 \text { cases }\end{array}$ \\
\hline
\end{tabular}




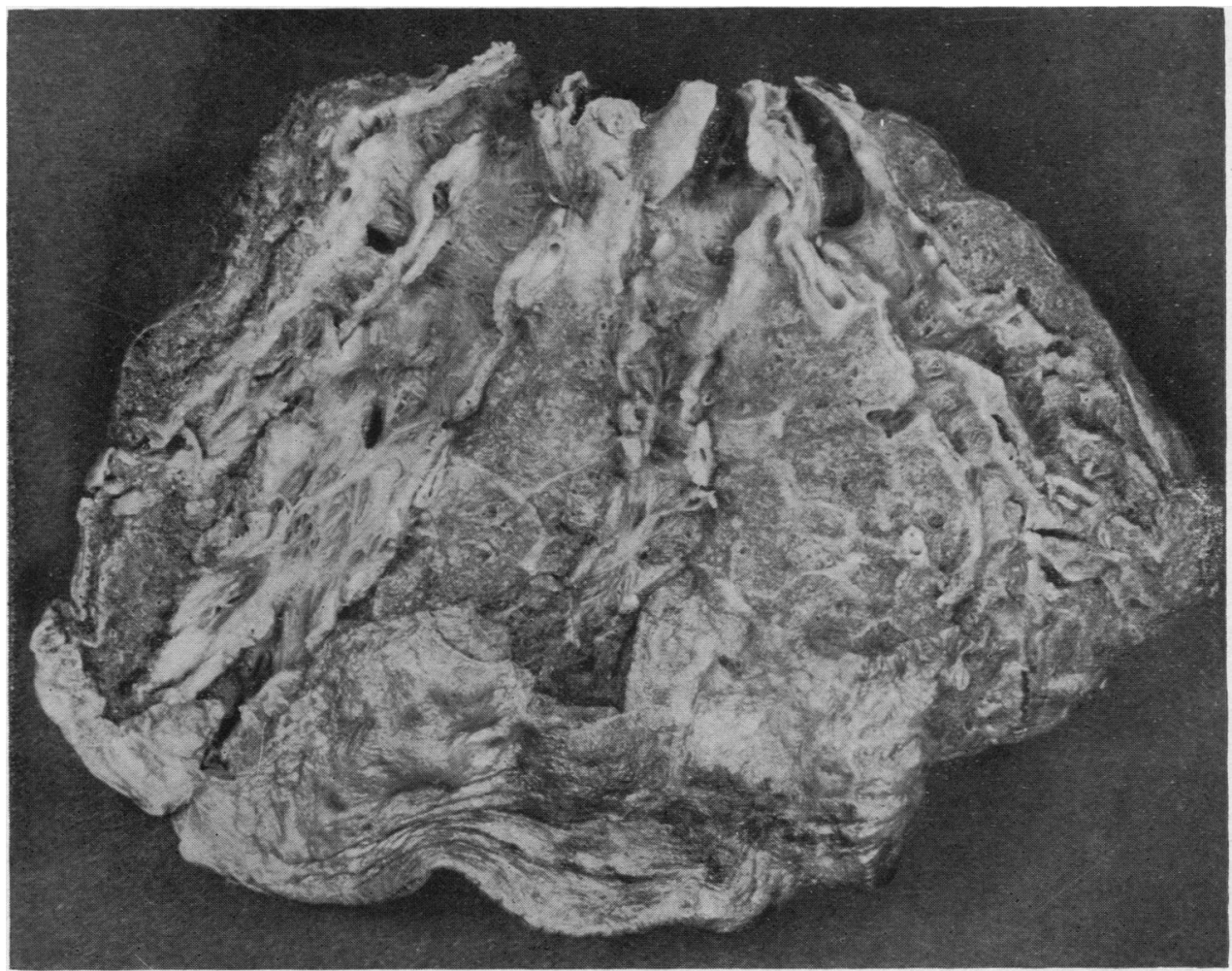

Fig. 6.-Operation specimen from the patient whose bronchogram is shown in Fig. 4. Aerated lung is apparent between the diseased bronchi which terminate just beneath the pleura, although only four side branches from the posterior basal bronchus can be seen. The anterior, middle, and posterior basal bronchi are shown.

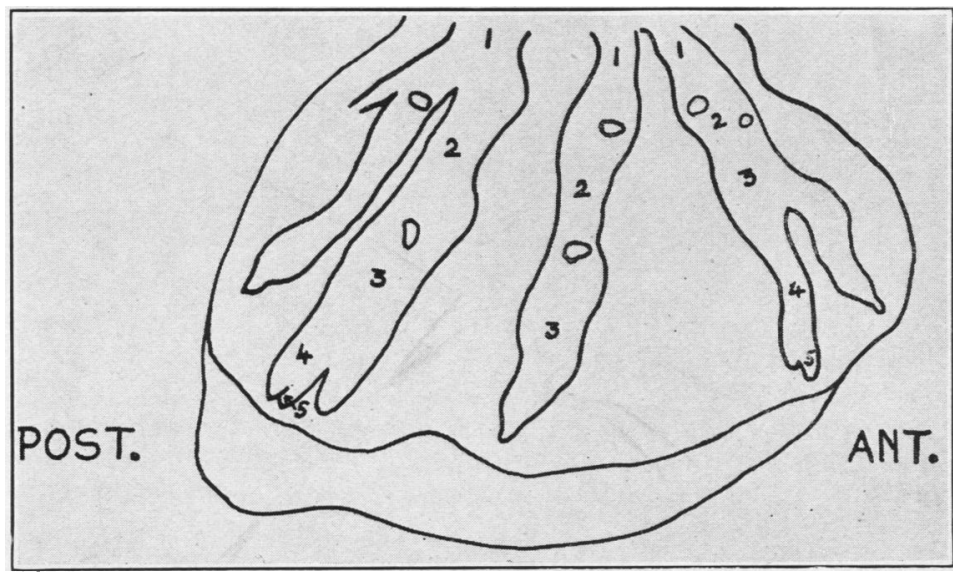

Fig. 7.-Diagram to indicate the number of branches arising from the posterior middle and anterior basal bronchi in the specimen shown in Fig. 6. 


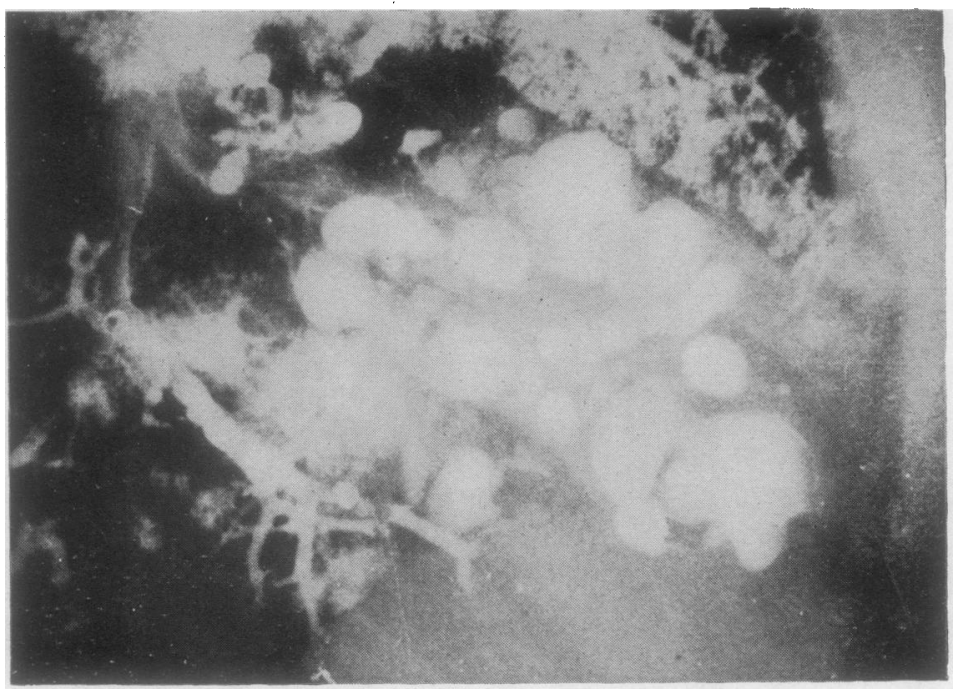

Fig. 8.-Bronchogram showing "saccular" bronchiectasis of right middle lobe. The continuity between the circular areas of lipiodol filling is not always obvious but it suggests only three or four branches. The valvular appearance of the bronchial walls seen in the macroscopic specimen accounts for the loculated appearance of the lipiodol.

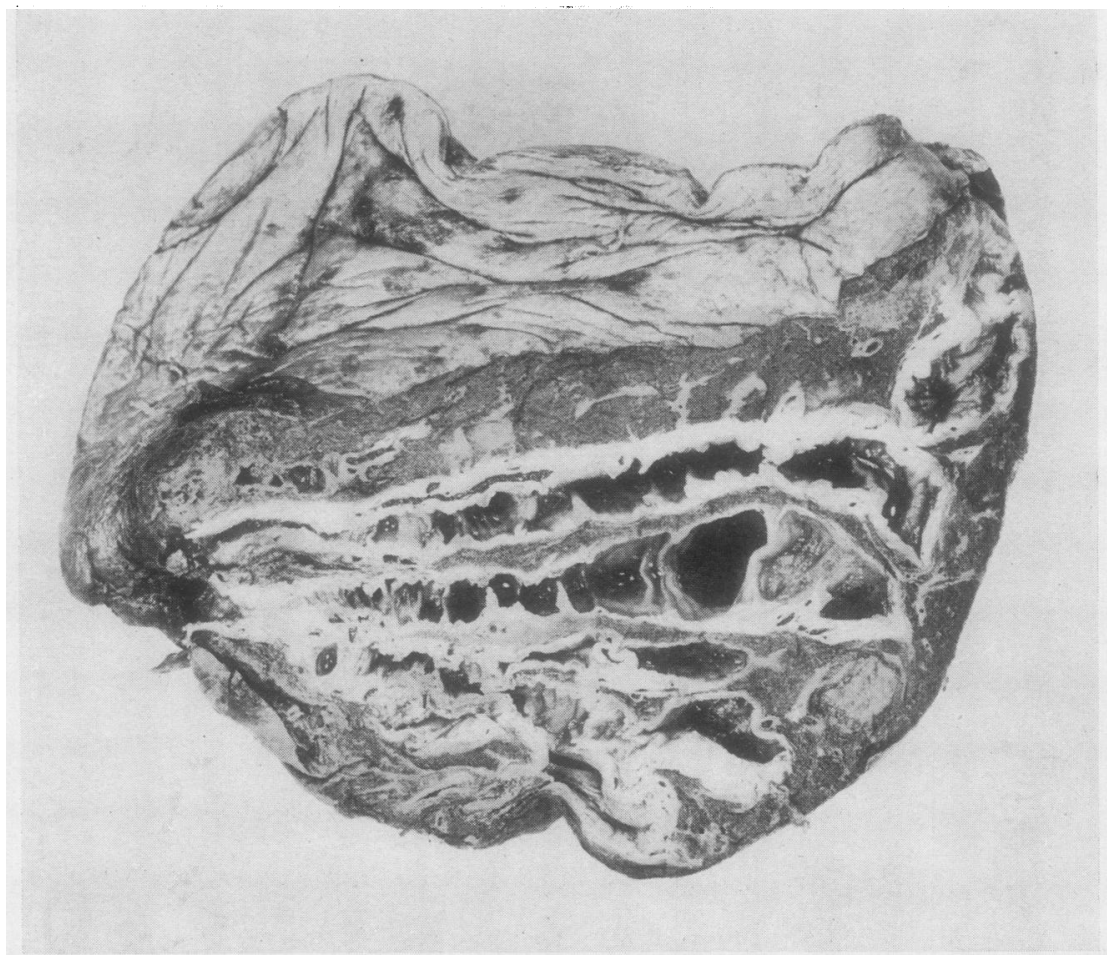

FIG. 9.-Operation specimen of the right middle lobe seen in the bronchogram in Fig. 8. The increasing diameter of the bronchi as they pass to the periphery is well seen. This terminal part represents the fourth generation of the bronchial tree. 
of the specimen. In many cases, although the peripheral part of the bronchial tree can be traced by dissection, it is found to be plugged completely with a cast of thick yellow sputum. The cellular infiltration and swelling of the wall which are also frequently seen in the bronchial and bronchiolar wall may further help to impede filling of these branches with lipiodol. These factors operate both in the first and second groups.

In the second group a third factor also operates, namely obliteration of the bronchial lumen, although frequently remnants of the larger bronchi can be seen beyond the limits of the patent bronchial tree. Some of the bronchi terminate abruptly in a bed of fibrous tissue which passes into the lung in the direction of the long axis of the bronchus as a discrete cord, firm and white macroscopically. In cross-section bundles of muscle fibres, elastic tissue, and a capillary network are seen, with, on occasion, plates of cartilage (Fig. 10). In some of these remnants of

FIG. 10.-Microscopic section to show the continuation of the posterior basal bronchus seen in Fig. 6. Several small lumina, which are continuous with the main bronchus, are seen in a bed of fibrous tissue containing also muscle fibres and elastic tissue whose presence was confirmed by special stains.

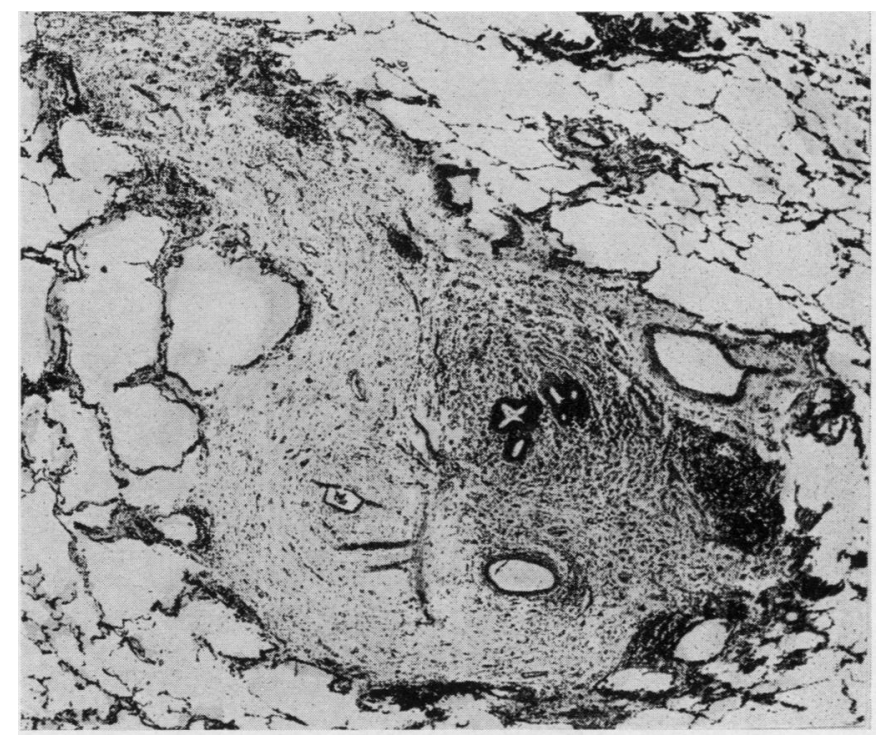

epithelium reappear to line cysts which are filled with material which stains with Mayer's mucicarmine stain and is usually quite free of cells. Some are macroscopically visible in the scar, and even more can be demonstrated by serial microscopic sections.

In the examples of the third group which have been examined histologically remnants of the bronchial tree have not been demonstrated to be directly continuous with the dilated bronchi (Figs. $11 a$ and $b$ ).

\section{Discussion}

The classification of bronchiectasis is still in a primitive stage, as it is usually based on the impression which the shape of the diseased bronchus makes on the observer. For example, one group is compared to a bunch of grapes, thus carrying on an ancient tradition in which pathological conditions are described in gastro- 


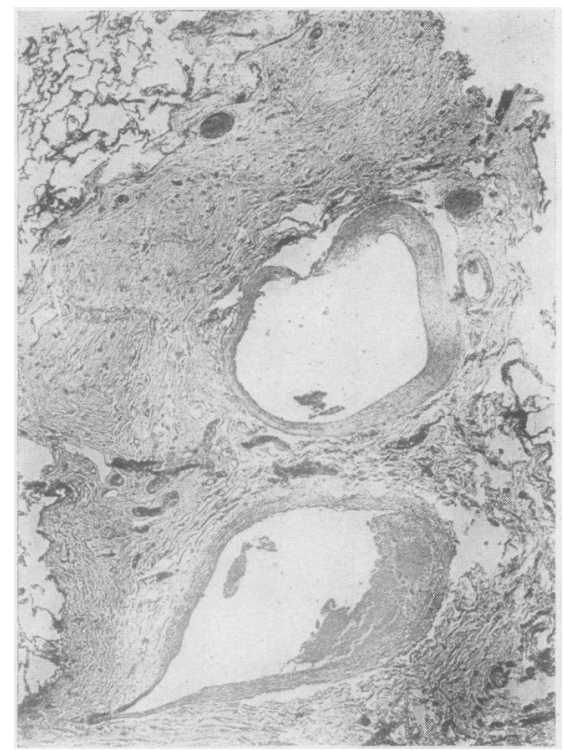

nomical terms. Although picturesque, there is little value in such terminology as an indication of aetiology or pathogenesis, and, moreover, there is lack of general agreement in its usage.

Terminology.-Ewart, in Allbutt's $A$ System of Medicine, published in 1898, describes three types of bronchiectasis: (1) Regular or cylindrical ; (2) fusiform ; (3) globular or sacculated. He mentions a modification of the third group which is a " bead-like" variety.

Since the introduction of lipiodol injection of the bronchial tree by Sicard and Forestier in 1923, most authors describe the different types of bronchiectasis according to the shape of the bronchi seen in the bronchogram. For example, Moll (1932), considering these very similar to those seen at necropsy,

FIG. $11(a$ and $b)$. -11 a shows in cross-section a fibrous cord continuous with the termination of a cartilaginous bronchus. Two large blood vessels are seen but no remnants of bronchial epithelium can be identified. In $11 b$ is shown a cystic space limed with epithelium into which the cord expanded.

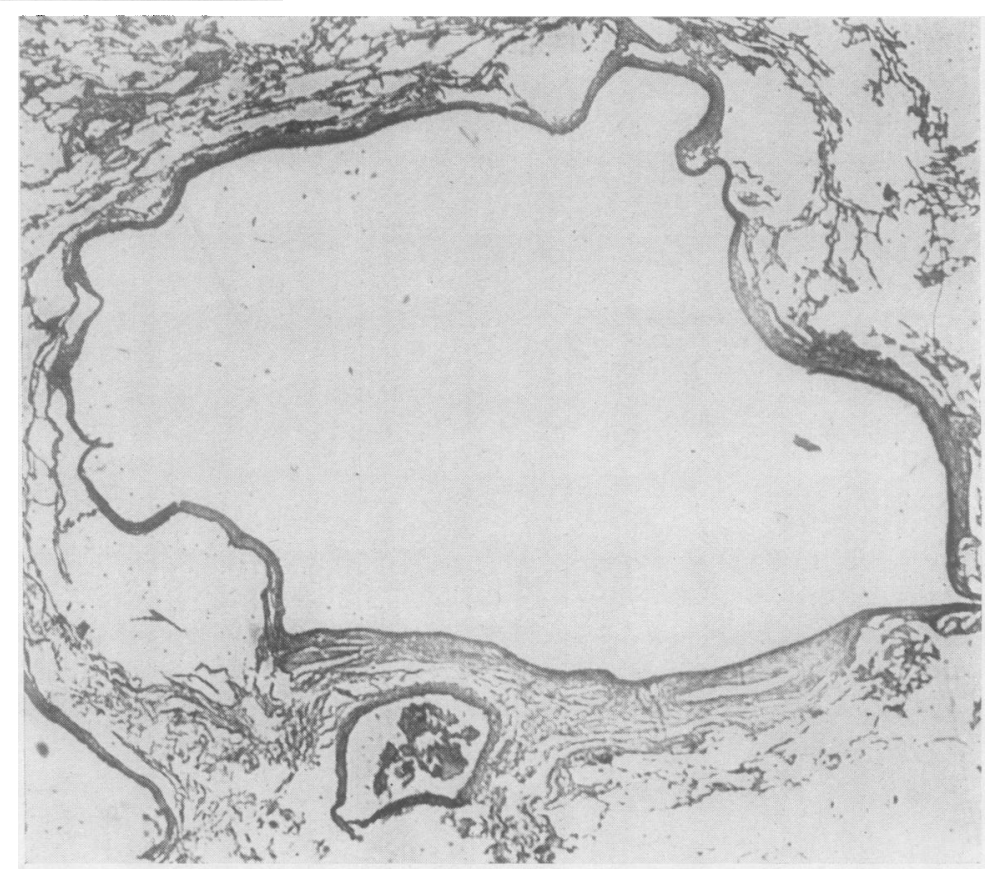

classifies them as follows: (1) Uniform, tubular, or cylindrical ; (2) fusiform or glove finger ; (3) globular or sacculated ; (4) moniliform or bead-like.

In addition he considers there are intermediate forms between tubular and sacculated types. In the moniliform the dilatation "occurs along the course of 
the bronchi and near the bifurcation of the secondary bronchial branches " (Moll, 1932). It resembles Group I of this series in the integrity of the peripheral subdivisions, but in one example examined histologically the structure of the bronchial wall is different from that usually seen in cylindrical bronchiectasis, suggesting a variation in pathogenesis.

In their series of cases studied to assess the prognosis of the condition, Perry and King (1940) divided them into two groups, the "cylindrical" and the "saccular or cystic," and found that the latter carried a graver prognosis.

Classification of the different types of bronchiectasis according to the form and shape of the dilated bronchi is peculiarly elastic, as there are no objective criteria: rather does it depend on the interpretation of each observer. For example, dilatation described by Findlay and Graham in 1927 as "tubular" corresponds to Moll's second or "fusiform " group rather than to his first, " cylindrical or tubular" one. Further evidence of confusion in criteria used in classification is the use by many authorities of some or all of the following terms as interchangeable ; saccular, cystic, congenital, and atelectatic (Thorpe, 1929; Ballon, Singer, and Graham, 1931 ; Roberts and Nelson, 1933 ; Arc, 1936).

Furthermore, it is difficult for even one group of observers to segregate all cases into rigid categories. Illustrative of this is an article of Roles and Todd (1933) in which they divide cases of bronchiectasis into five groups. Even the names applied to these (tubular, early fusiform, late fusiform commencing sacculation, fusosaccular, saccular, or ? congenital) indicate that the territory of one extends into that of another. Other names, such as "ampullary" used by Sicard and Forestier (1932) could be added to this list of terms and more evidence of inconsistency produced, but this would only serve to increase confusion.

The groups used in this investigation correspond approximately to the first three of Moll. Such a grouping has been useful in a preliminary analysis of cases, as, in spite of this disparagement of a morphological classification of the condition, the form of the diseased bronchus is important, in so far as it is this which the clinician sees when he examines the bronchogram. It is from this that he will try to deduce the natural history of the disease and to assess its prognosis. Any significance which can be attached to the bronchogram will therefore have a clinical application.

Further papers are being prepared on the correlation between the radiological features and the natural history and pathology of the condition.

The Relation between the Type of Bronchiectasis and its Position in the Bronchial Tree.-Many authors have considered that the form of the dilatation is dependent on the type of bronchus involved. Ewart stated that, with the exception of the bead-like variety, saccular dilatations are always terminal. Moll also describes the saccular type as involving terminal bronchi, adding that it is rare to find any "continuation of the bronchus on the terminal side beyond the dilatation." Fleischner (1940) considers that the "cystic form" involves the smaller bronchioles and the "cylindrical type" the larger ones.

In such descriptions the authors have considered that proximity of a bronchiectatic bronchus to the pleura indicated that the finer bronchioles were involved. The 
observations reported here show that the termination of the diseased bronchus may be immediately below the pleura and yet represent only the fifth generation in the bronchial tree. The picture of the specimen used as an example of Group II (Fig. 6) shows well that only four subdivisions of the bronchial tree can be seen although the bronchus is traced to within a few millimetres of the pleura. The specimen shown in Fig. 9 illustrates this same point in a case of saccular bronchiectasis. Only by counting from the hilum can the position of a bronchus in the bronchial tree be determined. It is important therefore that any disease process be localized not merely by its proximity to the hilum or pleura but by its position in the progressive subdivision of the bronchial tree. It is possible that where there has been much destruction and deformation of the proximal bronchi this may not be dependable ; but, in this instance, the regularity of the proximal part of the bronchus and the normal appearance of the openings to the side branches, which can be seen in the illustration, preclude this.

Roles and Todd have taken "tubular" to represent the condition where terminal bronchi do not taper in the normal way. In their cases, which are described as " cylindrical" by most authors, the terminal bronchi did not fill. That cylindrical bronchiectasis does involve the large bronchi, as described by Fleischner and others, is true ; but these are usually the only ones which show in a bronchogram and this does not mean that the peripheral branches are untouched by the disease process. Examination of the specimen shows that the contrary is true.

It may be argued that in the "cylindrical" type the occlusion of the peripheral bronchi and bronchioles by sputum as described here may be a temporary feature produced by stasis developing during lobectomy. But the constant failure of these cases to show satisfactory filling with lipiodol, even after posturing, suggests that this blockage is a constant state in the condition. Functionally, then, these peripheral bronchioles are abnormal, although anatomically they may be little distorted. In the other types the failure to fill is due to obliteration of the bronchial lumen, so that even where a cystic remnant of a bronchus may be demonstrable it is no longer in continuity with the trachea.

\section{SUMMARY}

The varying reduction in the number of subdivisions of the bronchial tree seen in bronchograms of bronchiectasis has been investigated in 45 cases. Counts have been made of the number of bronchial subdivisions present in the posterior basal broncho-pulmonary segment by examining the bronchograms, operation specimens, and microscopic serial sections. Counts made on three normal lungs have been used as controls.

Preliminary grouping of the specimens on the morphological appearance of the bronchograms was made, and there was shown to be correlation between this and the degree of reduction of bronchial subdivision. In the first or "cylindrical" group, the number of branches which could be identified in the specimens was within normal limits, although fewer bronchi than is normal were seen in the bronchogram. This failure to fill with lipiodol is often associated with plugging of the peripheral branches by secretion. The number of bronchi seen in the second or "varicose" group was reduced in both the radiographs and the specimens. A complete bronchial 
tree could not be identified in these cases, although the larger bronchi frequently persisted as discrete cords beyond the point at which the bronchial lumen was obliterated by fibrous tissue. Such remnants were not usually present in the third or "saccular" group, which showed least bronchi of any of the groups, as even on microscopic examination only four generations of the bronchial tree could be demonstrated.

Because in this type the dilated bronchi are usually subpleural, "cystic" bronchiectasis has generally been considered to involve the terminal bronchioles; but by counting the number of generations of the bronchial tree present between the hilum and the cysts, it has been shown that they usually represent proximal divisions.

The present terminology and classification of bronchiectasis are discussed.

I am indebted to Dr. E. S. J. King (pathologist, Royal Melbourne Hospital) and Mr. John I. Hayward (thoracic surgeon, Royal Melbourne Hospital) for their unfailing encouragement and advice in this work. The illustrations were prepared by Mr. R. Inglis, hospital photographer, and Mr. Rosenthal, of the Visual Aids Department, Melbourne University.

Arce, J. (1936-7). J. thorac. Surg., 6, 344.

Ballon, H., Singer, J. J., and Graham, E. A. (1931-2). Ibid., 1, 154, 296, 397, 502.

Brock, R. C. (1946). The Anatomy of the Bronchial Tree. London.

Broman, I. (1923). Anat. Anz., 57, 95.

Ewart, W. (1898). In A System of Medicine by many writers, ed. by T. C. Allbutt. Vol. 5, p. 58. London.

Findlay, L., and Graham, S. (1927). Arch. Dis. Childh., 2, 71.

Fleischner, F. (1940). Amer. Rev. Tuberc., 42, 297.

Jennings, G. H. (1937). Brit. med. J., 2, 963.

Moll, H. H. (1932). Quart. J. Med., 25, 457.

Palmer, D. M. (1936). Amer. J. Anat., 58, 59.

Perry, K. M. A., and King, D. S. (1940). Amer. Rev. Tuberc., 41, 531.

Roberts, J. E. H., and Nelson, H. P. (1933). Brit. J. Surg., 21, 277.

Roles, F. C., and Todd, G. S. (1933). Brit. med. J., 2, 639.

Sicard, J. A., and Forestier, J. (1923). Pr. méd., 31, 493.

- (1932). The Use of Lipiodol in Diagnosis and Treatment. London.

Thorpe, E. S. (1929). Amer. J. med. Sci., 177, 759. 